\title{
Robotic radical cystectomy in bladder cancer: is it the future?
}

This article was published in the following Dove Press journal:

Open Access Surgery

24 May 2014

Number of times this article has been viewed

\author{
Abdullah Erdem Canda' \\ Ali Fuat Atmaca' \\ Muhammed Ersagun Arslan² \\ Murat Keske 2 \\ Ozer Ural Cakici ${ }^{2}$ \\ Serdar Cakmak ${ }^{2}$ \\ Davut Kamaci \\ Emre Urer ${ }^{2}$ \\ 'Department of Urology, School of \\ Medicine, Ankara Ataturk Training \\ and Research Hospital,Yildirim \\ Beyazit University, Ankara, Turkey; \\ ${ }^{2}$ Department of Urology, Ankara \\ Ataturk Training and Research \\ Hospital, Ankara, Turkey
}

\begin{abstract}
Open radical cystectomy $(\mathrm{RC})$ is the gold standard surgical approach in the management of muscle invasive bladder cancer in addition to high-grade, recurrent, noninvasive tumors. With the development of surgical robotic technology, robotic-assisted laparoscopic radical cystectomy (RARC) is increasingly being performed as a minimally invasive surgical approach. A learning curve exists with a recommended case number of at least 20 RARC procedures in order to achieve satisfying outcomes in terms of operation time, complication rate, and oncological outcomes, including positive surgical margins (SMs) and lymph node (LN) yield. In the current literature, long-term outcomes of RARC are not yet available. Due to the outcomes of the published literature, RARC seems to have satisfactory oncologic and functional outcomes in addition to acceptable complication rates. Intraoperative blood loss and transfusion rates seem to be decreased in RARC series when compared to open approaches. On the other hand, a number of authors have reported decreased complication rates but increased operation time in the robotic approach. Similar oncologic results including positive SM rates and LN yields were detected in most comparative publications. Totally intracorporeal RARC with urinary diversion is a complex procedure and the number of centers performing this type of surgery is currently very limited. Although, it is still too early to make strict conclusions about RARC, RARC with intracorporeal urinary diversion has the potential to be the future of robotic bladder cancer surgery. Therefore, further prospective and randomized studies with increased numbers of patients and with longer follow-up are needed. Lastly, RARC may be related to increased cost when compared to open surgery, although controversial reports exist about this issue.
\end{abstract}

Keywords: intracorporeal urinary diversion, outcomes, open versus robotic, robotic surgery

\section{Introduction}

Robotic-assisted laparoscopic radical cystectomy (RARC) is increasingly being performed in the management of invasive bladder cancer as a minimally invasive surgical approach, although open radical cystectomy (RC) is still the gold standard surgical approach in the management of muscle invasive bladder cancer, in addition to high-grade, recurrent, noninvasive tumors. ${ }^{1}$

Herein, we reviewed the current literature regarding RARC including its learning curve, oncologic outcomes, functional outcomes, complications, open versus robotic approach, RARC with intracorporeal urinary diversion, and its cost.

\section{Learning curve and RARC}

An edited video of the RARC procedure performed by Canda can be watched here (Supplementary video). RARC is a complex procedure that includes three steps, 
including neurovascular bundle (NVB)-sparing RARC, bilateral extended pelvic lymph node (LN) dissection, and urinary diversion. In most of the published series, the first two steps are performed with the surgical robot and the last step (urinary diversion) is performed extracorporeally. ${ }^{2}$

In order to reach an operative time of 6.5 hours, it has been suggested that performing 20 RARC procedures was required, whereas in order to obtain an LN yield of 20 or more, 30 cases are required. ${ }^{3}$ In other publications, 20-40 cases were suggested to complete the learning curve of RARC. ${ }^{4}$ On the other hand, Hayn et al reported that initial experience with RARC did not affect the incidence of positive surgical margins (SMs), operative and postoperative complications, and overall survival, including a series of 164 cases. ${ }^{5}$ They reported that case number was significantly associated with shorter operative time and mean number of LNs retrieved. ${ }^{5}$

In a multicenter study by the International Robotic Cystectomy Consortium (IRCC) that included a series of 496 RARC cases by 21 surgeons at 14 institutions, the learning curve for RARC demonstrated an acceptable level of proficiency following performing 30 procedures for proxy measures of RARC quality. ${ }^{6}$ However, it is important to keep in mind that learning curves for RARC are subjective and based on nonvalidated metrics. ${ }^{7}$

In summary, at least 20 procedures are suggested to be performed in order to complete the learning curve of RARC.

\section{Oncologic outcomes of RARC}

LN yield and SMs in RC are considered to be the most important parameters in surgical oncologic quality and efficacy. A positive $\mathrm{SM}$ rate of $<10 \%{ }^{8,9}$ and an $\mathrm{LN}$ yield of $>15 \mathrm{LNs}^{10-12}$ are recommended for oncological sufficiency in open $\mathrm{RC}$.

Positive SMs were detected in 67 (4.2\%) of 1,589 patients who underwent open $\mathrm{RC}$ as reported by the Memorial Sloan-Kettering Cancer Center (New York, NY, USA). In the 5 years after cystectomy, the rates of disease-specific survival for the negative and positive SM groups were $72 \%$ and $32 \%$, respectively. On multivariate analysis, tumor stage, positive SMs, vascular invasion, presence of positive LNs, LN yield, and the number of positive nodes were found to be associated with disease-specific death. ${ }^{13}$ The same team reported factors that influence bladder cancer outcomes following RC. Five-year postcystectomy survival and local recurrence rates were reported to be $54 \%$ and $15 \%$, respectively. The outcomes of 2,091 patients who underwent RC from the Mayo Clinic with a median postoperative follow-up of 16.6 years were reported recently. Late recurrence was identified in 82 patients (3.9\%). On multivariate analysis, younger age, non-muscle-invasive disease, and prostatic urethral involvement were detected to be significantly associated with an increased risk of late recurrence. On multivariate analysis, younger patient age, muscle invasive disease, and nonurothelial recurrence site but not time to recurrence were detected to be associated with a significantly increased risk of death from bladder cancer following recurrence after RC. ${ }^{14}$

The oncologic outcomes of the selected published RARC series including the IRCC outcomes are presented in Table 1 in terms of positive SM rates and LN yields. ${ }^{15-20}$ The mean LN yield ranges between 15-21 and the positive SM rate ranges between $1.4 \%-7 \%$; these suggest that RARC has similar results to open surgery. ${ }^{15-20}$

In an IRCC study that included 527 patients from 15 institutions between 2003 and 2009, tumor stage, sequential case number, institution volume, and surgeon volume were detected to be significantly associated with the likelihood of undergoing LN dissection. On multivariate analysis, surgeon volume was identified to be the most statistically significantly associated factor related with $\mathrm{LN}$ dissection. The IRCC defined high-volume surgeons as robotic surgeons who have performed more than 20 cases. The study also detected that high-volume surgeons were almost three times more likely to perform LN dissections than lower volume surgeons. ${ }^{19}$

Another interesting IRCC study evaluated if previous robot-assisted radical prostatectomy (RARP) experience has an impact on RARC outcomes. ${ }^{20}$ Overall, 496 RARC patients were included by 21 surgeons at 14 centers. They divided surgeons into four groups according to their previous RARP experience as $\leq 50$, 51-100, 101-150, and $>150$ cases. The operative time, blood loss, LN yield, and

Table I SM rates and LN yields in the published literature

\begin{tabular}{|c|c|c|c|c|c|c|}
\hline Reference & Institution & Country & Year & $\mathbf{N}$ & SM rate & LN yield (range) \\
\hline Tyritzis et al ${ }^{15}$ & Karolinska Institutet & Sweden & 2013 & 70 & $1.4 \%$ & $20.9 \pm 9.4(7-52)$ \\
\hline Xylinas et $\mathrm{al}^{16}$ & Weill Cornell Medical College & USA & 2013 & 175 & $5 \%$ & $19(12-28)$ \\
\hline Treiyer et al ${ }^{17}$ & Saarland University & Germany & 2012 & 91 & $2.1 \%$ & $15(4-33)$ \\
\hline Canda et $\mathrm{al}^{18}$ & Ankara Ataturk Hospital & Turkey & 2012 & 27 & $3.7 \%$ & $24.8 \pm 9.2(8-46)$ \\
\hline Hellenthal et $\mathrm{al}^{19}$ & IRCC & Multicenter study & 2011 & 527 & NR & $17.8(0-68)$ \\
\hline Hayn et $\mathrm{al}^{20}$ & IRCC & Multicenter study & 2010 & 482 & $7 \%$ & $17.8(0-68)$ \\
\hline
\end{tabular}

Abbreviations: IRCC, International Robotic Cystectomy Consortium; LN, lymph node; N, number of patients; NR, not reported; SM, surgical margin. 
increased pathologic stage were detected to be significantly associated with previous RARP experience. On the other hand, margin status was not significantly associated with previous RARP experience. ${ }^{20}$

In a recent study from the Karolinska Institutet (Solna, Sweden) evaluating the outcomes of 70 RARC procedures, Kaplan-Meier estimates for recurrence-free, cancer-specific, and overall survival at 24 months were reported to be $80.7 \%$, $88.9 \%$, and $88.9 \%$, respectively. ${ }^{15}$ Khan et al from the Guy's and St Thomas' National Health Service (NHS) Foundation Trust (London, UK) reported the long-term oncologic outcomes of 14 patients (eleven males and three females) upon whom they had performed RARC due to muscle-invasive or high-grade non-muscle-invasive or bacillus CalmetteGuérin-refractory carcinoma in situ disease with a follow-up of $\geq 5$ years. ${ }^{21}$ Overall survival of $64 \%$, disease-specific survival of $75 \%$, and disease-free survival of $50 \%$ were reported. Of the patients, five died during follow-up (three of metastatic disease and two of unrelated causes). Overall, six patients were alive and disease-free. ${ }^{21}$

Another interesting recent paper from the Mayo Clinic (Phoenix, AZ, USA) evaluated the outcomes of 50 RARC patients with LN-positive disease. ${ }^{22}$ The mean follow-up was 42 months. Of the patients, $34 \%$ had $\leq$ pT2 disease and $66 \%$ had pT3/T4 disease. During follow-up, 29 patients had recurred, and 22 patients had died of disease. The mean time to recurrence was 10 months. The estimated overall survival times at 36 and 60 months were $55 \%$ and $45 \%$, respectively. The recurrence-free survival times at 36 and 60 months were $43 \%$ and $39 \%$, respectively. ${ }^{22}$

In summary, RARC has acceptable oncologic outcomes in terms of SMs and LN yield as seen in the published literature.

\section{Functional outcomes of RARC}

Functional outcomes include urinary continence and erectile function following RARC. In the published literature, only a few authors have reported functional outcomes following RARC. Menon et al stated that NVB-sparing RARC combines the oncological principles of open surgery with the technical advantages of the surgical robot, which allows precise, gentle, quick, and safe surgery. ${ }^{23}$ Therefore, robotic surgery seems to give the advantage of better preserving the NVBs, which is expected to have an impact on postoperative functional outcomes. Figure 1 shows abdominal port locations and Figure 2A-D show NVB-sparing RARC.

Due to the results from the Karolinska Institutet, 70 patients were operated on by two experienced

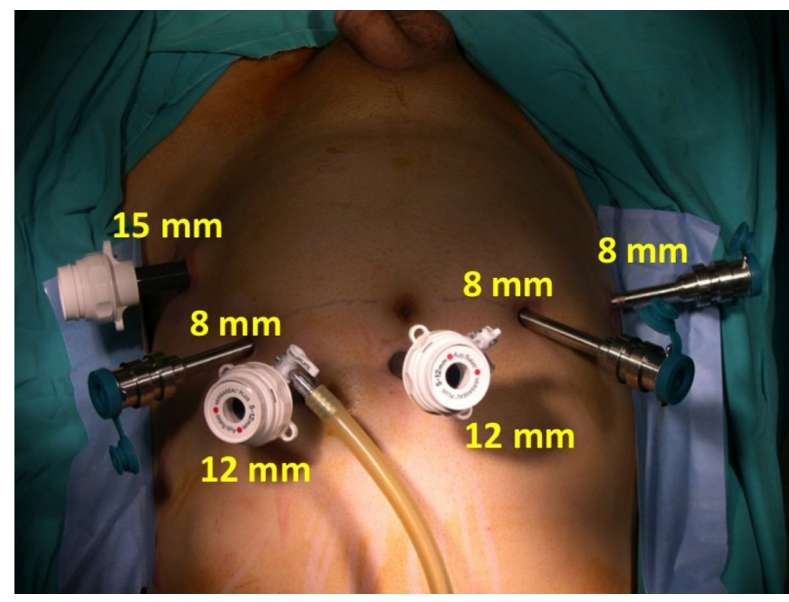

Figure I Abdominal port locations for RARC.

Note: Image from surgical archive of Canda.

Abbreviation: RARC, robotic-assisted laparoscopic radical cystectomy.

robotic surgeons. ${ }^{15}$ RARC with totally intracorporeal modified Studer ileal neobladder formation was performed. Daytime continence and satisfactory sexual erectile function at 1 year were reported at $70 \%$ and $90 \%$, respectively. ${ }^{15}$ Guy's and St Thomas' NHS Foundation Trust Hospital, which was one of the first European urology centers that introduced RARC, reported its outcomes on 14 patients who completed a minimum of 5 years and a maximum of 8 years of follow-up following RARC and extracorporeal urinary diversion. ${ }^{21}$ Ileal conduit $(n=12)$ or orthotopic neobladder $(n=2)$ were performed extracorporeally. They reported that four of four previously potent patients having NVB-sparing RARC recovered in terms of erectile function. ${ }^{21}$

Torrey et al reported the functional outcomes of 34 patients who underwent RARC with Indiana pouch continent cutaneous urinary diversion reconstruction at The City of Hope Cancer Center (Duarte, CA, USA). Of the available 31 patients, 30 patients (97\%) had daytime and nighttime continence at a mean follow-up of 20.1 months. ${ }^{24}$

The mean follow-up was 6.3 (standard deviation of 2.9; range of 1.8-11.3) months in a Turkish study on 23 patients who underwent RARC and intracorporeal Studer pouch reconstruction (21 males, two females). Overall, 21 patients underwent bilateral NVB-sparing RARC, one unilateral NVB-sparing RARC, and one non-NVB-sparing surgery. Among the 21 men, 15 were available for postoperative urinary continence evaluation. Of those, eleven (73.3\%) were fully continent during the daytime and four $(26.6 \%)$ had mild urinary incontinence. ${ }^{18}$

In summary, RARC seems to have promising functional outcomes, although to date, very few centers have reported functional outcomes related to their RARC experience. 

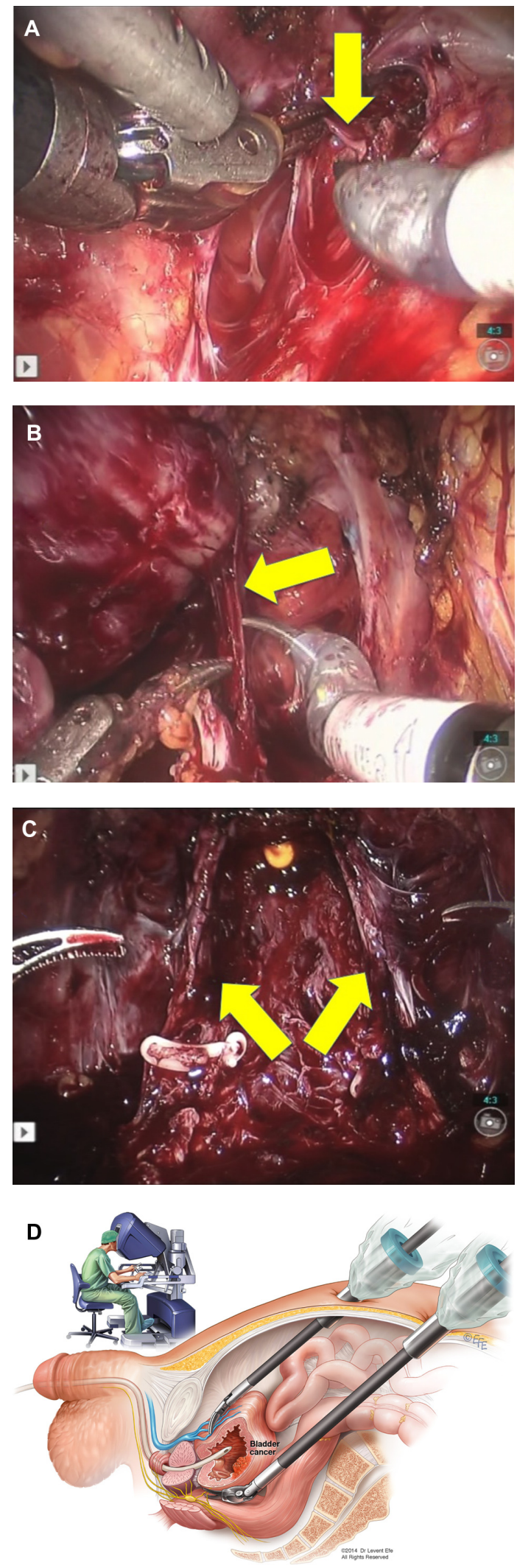

Figure 2 (A) High anterior release neurovascular bundle-sparing (arrow, left side); (B) appearance preserved right neurovascular bundle (arrow); (C) appearance of bilaterally preserved neurovascular bundles in the pelvis (arrows); (D) artwork of robotic neurovascular bundle-sparing RARC.

Notes: Images A-C from the surgical archive of Canda; Figure D drawn by Levent Efe, medical illustrator.

Abbreviation: RARC, robotic-assisted laparoscopic radical cystectomy.

\section{Complications of RARC with extracorporeal urinary diversion}

In most of the publications reporting the complications of RARC, the modified Clavien-Dindo grading system was used to classify complications, including perioperative (30-day) and within 31-90 days of surgery (Table 2). ${ }^{25,26}$

Shabsigh et $\mathrm{al}^{27}$ from the Memorial Sloan-Kettering Cancer Center reported on the type, incidence, and severity of early postoperative morbidities following $\mathrm{RC}$ using a standardized reporting methodology. Overall, they included 1,142 cases in their study. Of these patients, $64 \%$ experienced a complication within 90 days of surgery and of those, $67 \%$ experienced a complication during the operative hospital admission and $58 \%$ following discharge. Grade 0 , grade $1-2$, and grade $3-5$ complications were detected at rates of $36 \%$, $51 \%$, and $13 \%$, respectively. Gastrointestinal, infectious, and wound-related complications were the most common complications detected, at rates of $29 \%, 25 \%$, and $15 \%$ of the cases, respectively. The 30 -day mortality rate was $1.5 \%$. The authors concluded that accurate reporting of postoperative complications after $\mathrm{RC}$ is essential for counseling patients, combined modality treatment planning, clinical trial design, and assessment of surgical success. ${ }^{27}$

In a multi-institutional study including 277 patients with RARC performed in four institutions, complications occurred in 68 patients $(30 \%)$, with $7 \%$ having Clavien grade $\geq 3$ complications. On multivariate analysis, decreased age and increased American Society of Anesthesiologists (ASA) score were detected as predictors of higher Clavien complication score. ${ }^{28}$

In another series of 34 RARC patients with Indiana pouch continent cutaneous urinary diversion reconstruction, 175 complications following RARC were identified in $32(94 \%)$ of 34 patients. Within 90 days of surgery, 31 (91\%) of 34 patients experienced $\geq 1$ early complication.

Table 2 Modified Clavien classification of complications

\begin{tabular}{ll}
\hline Grade & Description \\
\hline I & $\begin{array}{l}\text { Any deviation from the normal postoperative course } \\
\text { without the need for pharmacologic treatment or } \\
\text { surgical, endoscopic, and radiologic interventions } \\
\text { Requiring pharmacologic treatment with drugs other } \\
\text { than such allowed for grade I complications (including } \\
\text { II }\end{array}$ \\
& $\begin{array}{l}\text { bequiring surgical, endoscopic, or radiologic intervention } \\
\text { III }\end{array}$ \\
IIIa & Intervention not under general anesthesia \\
IIIb & Life-threatening complication requiring intensive care \\
IV & unit management \\
V & Death of a patient \\
\hline
\end{tabular}


Of the 34 patients, 15 (44\%) reported $\geq 1$ late complications (>90 days). Most of the early and late complications were graded as minor. The most commonly identified complication in both intervals was infection detected in $22 \%$ and $37 \%$ of patients with early and late complications, respectively. ${ }^{24}$ In a series of 175 patients with RARC from the Weill Cornell Medical College (New York, NY, USA), early ( $<30$ days) and late (30-90 days) surgery-related complications were detected in 74 (42\%) and 59 (34\%) of patients, respectively. The perioperative mortality rate was $2.8 \%$. Four patients $(2.3 \%)$ required conversion to open surgery because of difficulty to progress. ${ }^{16}$

Nazmy et al evaluated perioperative complications stratified by urinary diversion type following RARC in 209 patients. Complications occurred in $77.5 \%$ of the patients within 90 days and of those, $32 \%$ were major complications. Most of the complications were related to the gastrointestinal, infectious, and hematological systems. Multivariate analysis revealed that patients with ileal conduit diversion had a decreased incidence of complications compared to patients with Indiana pouch and orthotopic bladder substitute diversion. ${ }^{29}$

In a series of 241 RARC patients, $80 \%$ had a complication of any grade $\leq 90$ days after robotic surgery. Overall, 475 adverse events (113 major, 362 minor) were identified postoperatively. Of these, 365 (77\%) occurred during the perioperative period (0-30 days). Within the first 90 days after surgery, 68 (35\%) patients experienced a major complication. Blood transfusions (43.9\%), infectious (16.2\%), gastrointestinal (14.1\%), and procedural (10.3\%) complications were the most common. Age, presence of comorbid diseases, preoperative hematocrit, estimated blood loss, and duration of surgery were identified as predictive factors of a complication of any grade. The presence of comorbid diseases, preoperative hematocrit, and orthotopic diversion were identified as predictive factors of major complications. ${ }^{30}$ In another study, the presence of preoperative renal insufficiency and intraoperative intravenous fluids of $>5,000 \mathrm{~mL}$ were significant risk factors associated with postoperative complications of any grade following multivariate analysis. For high-grade complications, significant independent risk factors included patient age of $\geq 65$ years, operative blood loss of $\geq 500 \mathrm{~mL}$, and intraoperative intravenous fluids of $>5,000 \mathrm{~mL} .^{31}$

In the IRCC multicenter study including 939 patients, $41 \%(n=387)$ and $48 \%(n=448)$ of patients had a complication within 30 and 90 days of robotic surgery, respectively. Of these, $52 \%$ were grade $0,29 \%$ were grade $1-2$, and $19 \%$ were grade $3-5$. The most common complications were related to the gastrointestinal (27\%), infectious (23\%), and genitourinary (17\%) systems. Increasing patient age group, administration of neoadjuvant chemotherapy, and blood transfusion were identified as independent predictors of any and high-grade complications on multivariate analysis. Thirty- and 90-day mortality were reported as $1.3 \%$ and $4.2 \%$, respectively. ${ }^{32}$

In summary, RARC appears to have acceptable complications rates as seen in the published literature.

\section{Open versus robotic approach}

The number of published studies evaluating open versus robotic RC is very limited. Mostly, published studies are retrospective, with limited numbers of patients. Only two papers were prospective, randomized studies; however, the number of patients included were very limited in both series. ${ }^{33,34}$ In addition, the follow-up time was short in almost all of these studies. Therefore, it was not easy to draw strict conclusions about the outcomes regarding open versus robotic RC comparison.

Regarding the initial prospective, randomized study by Nix et al, 21 patients were randomized to the robotic approach and 20 to the open surgery approach. ${ }^{33}$ The groups were similar in terms of patient characteristics. Significant advantages in favor of the robotic approach were found in terms of operating room time, estimated blood loss, time to flatus, time to bowel movement, and use of inpatient morphine sulfate equivalents. The overall complication rates and hospital stay length were similar between the groups. The mean LN yield was 19 in the robotic group and 18 in the open group. Regarding the second prospective, randomized trial by Parekh et al, 20 patients each were included in the robotic and open groups. ${ }^{34}$ Similar outcomes were detected in the two groups in terms of patient characteristics, operation time, postoperative complications, and final pathological stage. Oncologic outcomes, including positive SMs and mean LN yield were similar between the groups. ${ }^{34}$ On the other hand, estimated blood loss was significantly decreased in the robotic group. Although statistically not significant, excessive length of hospital stay ( $>5$ days) and transfusion rate were found to be decreased in the robotic group. ${ }^{34}$ Figure $3 \mathrm{~A}$ and B show robotic extended pelvic $\mathrm{LN}$ dissection.

Other retrospective series also reported their comparative outcomes related to open versus robotic RC. A summary of the selected publications on this subject is presented in Table 3. Similar outcomes were reported in terms of operation time in some studies, ${ }^{34,37,40}$ whereas others reported increased operation time in the robotic arm..$^{33,35,36,38}$ Decreased intraoperative blood loss ${ }^{33-36,38-40}$ and decreased transfusion rates $^{34-36,38}$ 

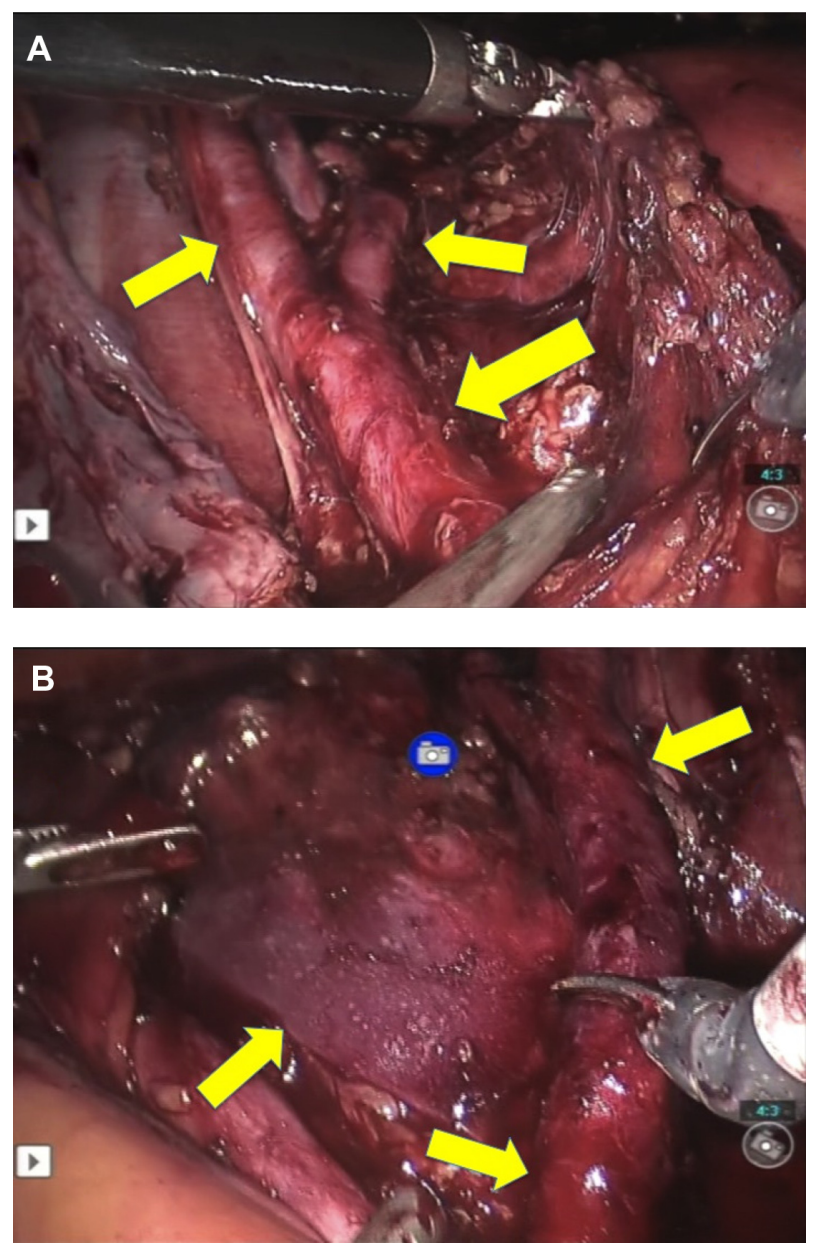

Figure 3 (A) Appearance of the skeletonized major vessels and extended pelvic extended lymph node dissection in the pelvis (arrows); (B) appearance of the skeletonized major vessels and extended pelvic extended lymph node dissection in the pelvis (arrows).

Note: Images from the surgical archive of Canda.

seem to be major advantages of the robotic approach in many

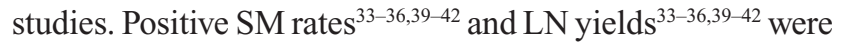
detected to be similar in most studies. Overall and major complications at postoperative 30 and 90 days were found to be significantly fewer in the robotic group in a prospective and randomized study by Ng et al. ${ }^{40}$ However, Parekh et al did not find any significant difference in terms of Clavien grade $\geq 2$ complications between the robotic and open groups. ${ }^{34}$ Likewise, many retrospective studies reported similar complication rates between open and robotic RC. ${ }^{33,34,36-38,41-43}$

Some of the other parameters that were evaluated in comparative studies included return of bowel function, time to resumption of regular diet, use of inpatient narcotics, and duration of hospital stay. Some authors reported quicker return of bowel function ${ }^{33,35}$ and decreased time to resumption of regular diet ${ }^{35,41}$ when using the robotic approach. The duration of hospital stay was reported to be decreased in the robotic group in some studies, ${ }^{34,35,40,41}$ whereas others reported similar data in both groups. ${ }^{33,36-39}$

The interim results of a randomized clinical trial comparing robotic versus open RC from the Memorial SloanKettering Cancer Center were presented at a late-breaking news plenary at the 2013 American Urological Association Congress (May 4-8, 2013; San Diego, CA, USA). ${ }^{44}$ A total of 116 patients were randomized to robotic or open cystectomy (59 robotic, 57 open). Patients undergoing robotic and open cystectomy experienced a mean 2.1 versus 1.7 complications, respectively, which was not found to be statistically significantly different. The operation time was longer for the robotic group (454 minutes vs 328 minutes); however, the estimated blood loss was less in the robotic arm. LN yield was similar in both groups ( 30 vs 25 ). Positive soft-tissue SM rates were also similar between the groups ( $3 \%$ robotic vs $5 \%$ open). Likewise, a similar length of hospital stay was detected in both groups (mean 8 days in both groups). They concluded that there was no difference in the rate or severity of complications within 90 days of surgery. ${ }^{44}$

Very recently, Leow et al evaluated the morbidity of robotic versus open $\mathrm{RC}$ in a contemporary population-based analysis in the US. We conducted a population-based, retrospective cohort study of patients who underwent RC at 279 hospitals across the US between 2004 and 2010. Overall, 34,672 open RC and 2,101 RARC patients were included. RARC use increased from $0.6 \%$ in 2004 to $12.8 \%$ in 2010 . Major complication rates (Clavien grade $\geq 3$ ) were similar between the open and robotic approaches $(7.0 \%$ vs $19.8 \%, P=0.2)$. The robotic approach had $46 \%$ decreased odds of minor complications (Clavien grade $1-2$; odds ratio [OR]: $0.54 ; P=0.03) .{ }^{45}$

In summary, intraoperative blood loss and transfusion rates seem to be decreased with similar oncologic results, including positive SM rates and LN yields in RARC series when compared to open approaches. On the other hand, RARC seems to have increased operation time but decreased complication rates compared to open surgery.

\section{RARC with intracorporeal urinary diversion}

The number of centers performing totally intracorporeal RARC including urinary diversion is currently very limited. In addition, the number of patients is very limited in published series when compared to RARC series with extracorporeal urinary diversion.

Due to the initial results from the Karolinska Institutet, 45 patients with RARC and intracorporeal neobladder reconstruction were pooled into three consecutive groups of 
Table 3 Outcomes of selected open versus robotic radical cystectomy publications

\begin{tabular}{|c|c|c|c|c|c|c|c|c|c|}
\hline \multirow[t]{2}{*}{ Ref } & \multirow[t]{2}{*}{ Study type } & \multirow[t]{2}{*}{$\mathbf{N}$} & \multirow[t]{2}{*}{ OR time } & \multirow[t]{2}{*}{ EBL } & \multirow[t]{2}{*}{ LOS } & \multirow[t]{2}{*}{ SM rate } & \multirow[t]{2}{*}{ LN yield } & \multicolumn{2}{|c|}{ Major complications } \\
\hline & & & & & & & & $0-30$ days & $31-90$ days \\
\hline \multirow[t]{4}{*}{33} & $P \& R$ & & & & & & & & \\
\hline & Robotic & 21 & $4.20 \mathrm{~h}$ & $258 \mathrm{~mL}$ & $5.1 \mathrm{~d}$ & $0 \%$ & 19 & 2.3 & \\
\hline & Open & 20 & $3.52 \mathrm{~h}$ & $575 \mathrm{~mL}$ & $6.0 \mathrm{~d}$ & $0 \%$ & 18 & 2.6 & \\
\hline & $P$-value & & $<0.0001$ & $<0.0001$ & NS & - & NS & NS & \\
\hline \multirow[t]{4}{*}{34} & $P \& R$ & & & & & & & & \\
\hline & Robotic & 20 & $300 \mathrm{~min}$ & $400 \mathrm{~mL}$ & $35 \%$ & $5 \%$ & II & $25 \%$ & \\
\hline & Open & 20 & $285 \mathrm{~min}$ & $800 \mathrm{~mL}$ & $10 \%$ & $5 \%$ & 23 & $25 \%$ & \\
\hline & $P$-value & & NS & 0.003 & $0.03^{a}$ & NS & NS & $N S^{b}$ & \\
\hline \multirow[t]{4}{*}{35} & Ret & & & & & & & & \\
\hline & Robotic & 58 & $7.8 \mathrm{~h}$ & $276 \mathrm{~mL}$ & $6.3 \mathrm{~d}$ & 7 & 21.3 & $24 \%$ & $20 \%$ \\
\hline & Open & 84 & $6.6 \mathrm{~h}$ & $\mathrm{I}, 522 \mathrm{~mL}$ & $10.8 \mathrm{~d}$ & 8 & 17.7 & $58 \%$ & $21 \%$ \\
\hline & $P$-value & & $<0.0001$ & $<0.0001$ & 0.004 & NS & NS & $<0.0001$ & NS \\
\hline \multirow[t]{4}{*}{36} & Ret & & & & & & & & \\
\hline & Robotic & 50 & $455 \mathrm{~min}$ & $350 \mathrm{~mL}$ & $9.5 \mathrm{~d}$ & $2 \%$ & 14.3 & 7I & - \\
\hline & Open & 100 & $349 \mathrm{~min}$ & $475 \mathrm{~mL}$ & $10.2 \mathrm{~d}$ & $1 \%$ & 15.2 & 136 & - \\
\hline & $P$-value & & $<\mathbf{0 . 0 0 0 I}$ & 0.02 & NS & NS & NS & NS & - \\
\hline \multirow[t]{4}{*}{37} & Ret & & & & & & & & \\
\hline & Robotic & 15 & $409 \mathrm{~min}$ & I,789 mL & $40 \mathrm{~d}$ & $9 \%$ & 21 & $6 \%$ & - \\
\hline & Open & II & $364 \mathrm{~min}$ & $657 \mathrm{~mL}$ & $37 \mathrm{~d}$ & $20 \%$ & 14 & $11 \%$ & - \\
\hline & $P$-value & & NS & 0.0015 & NS & NS & 0.04 & NS & - \\
\hline \multirow[t]{4}{*}{38} & Ret & & & & & & & & \\
\hline & Robotic & 35 & 578 & $448 \mathrm{~mL}$ & 29 & NR & 19 & $22 \%$ & \\
\hline & Open & 104 & 501 & $\mathrm{I}, 063 \mathrm{~mL}$ & 27 & NR & 13 & $77 \%$ & \\
\hline & $P$-value & & 0.008 & $<0.00 \mathrm{I}$ & NS & - & $<0.001$ & $N S^{c}$ & \\
\hline \multirow[t]{4}{*}{39} & Ret & & & & & & & & \\
\hline & Robotic & 36 & $338 \mathrm{~min}$ & $695 \mathrm{cc}$ & $7.9 \mathrm{~d}$ & 0 & 17 & NR & \\
\hline & Open & 29 & $398 \mathrm{~min}$ & I497 cc & $9.6 \mathrm{~d}$ & 0 & 14 & NR & \\
\hline & $P$-value & & 0.0002 & 0.0002 & NS & $N S^{d}$ & NS & - & \\
\hline \multirow[t]{4}{*}{40} & Ret & & & & & & & & \\
\hline & Robotic & 83 & $6.3 \mathrm{~h}$ & $460 \mathrm{~mL}$ & $5.5 \mathrm{~d}$ & $7.2 \%$ & 17.9 & $10 \%$ & $17 \%$ \\
\hline & Open & 104 & $5.9 \mathrm{~h}$ & 1172 mL & $8 d$ & $8.7 \%$ & 15.7 & $30 \%$ & $31 \%$ \\
\hline & $P$-value & & NS & $<\mathbf{0 . 0 0 0 I}$ & $<0.001$ & NS & NS & $0.007^{e}$ & $0.03^{e}$ \\
\hline
\end{tabular}

Notes: aPercentage of patients with length of stay $\leq 5$ days; ${ }^{b}$ percentage of patients with Clavien $\geq 2$ complications; ${ }^{c}$ percentage of patients with complications; ${ }^{\mathrm{d}}$ for pathologic T2 or lower disease; ${ }^{e}$ patients with major complications. Complications are classified due to modified Clavien classification. Bold $P$-values are statistically significant. Abbreviations: d, days; EBL, estimated blood loss; h, hours; min, minutes; LN, lymph node; LOS, length of hospital stay; N, number of patients; NR, not reported; NS, statistically not significant; OR time, operation time; P\&R, prospective and randomized; Ref, reference; Ret, retrospective; SM, surgical margin.

15 cases in each group in order to evaluate the complications according to the Clavien classification during the learning curve. The authors detected fewer complications in the groups over time, with a statistically significant decrease in late versus early complications. On the other hand, early Clavien grade 3 complications remained statistically significant (27\%) and did not decline over time. ${ }^{46}$ In a further study from the same team including 70 RARC patients with totally intracorporeal neobladder diversion, Clavien grade 3-5 complications were identified in 22 of 70 patients (31.4\%) at 30 days and 13 of 70 patients $(18.6 \%)$ at $>30$ days of surgery. At 90 days postoperative, the overall complication rate was $58.5 \%$. Clavien grade $<3$ and Clavien grade $\geq 3$ complications were detected in 15 of 70 patients $(21.4 \%)$ and 26 of 70 patients (37.1\%), respectively. ${ }^{15}$
In a series of 27 RARC patients with intracorporeal Studer pouch $(n=23)$, ileal conduit $(n=2)$, and extracorporeal Studer pouch $(n=2)$ reconstruction, nine minor (Grade 1 and 2) and four major (Grade 3-5) complications in the perioperative ( $0-30$ days) period; four minor and three major complications in the postoperative (31-90 days) period were reported..$^{18}$ An unusual complication was also reported by the same team of delayed massive transurethral hemorrhage due to external iliac artery pseudoaneurysm and ureteroiliac artery fistula following RARC and intracorporeal Studer pouch reconstruction in a 54-year-old male patient, which occurred 1 month after the surgery that was successfully managed by endovascular stenting. ${ }^{47}$ Figure 4A and B show intracorporeal Studer pouch reconstruction. 

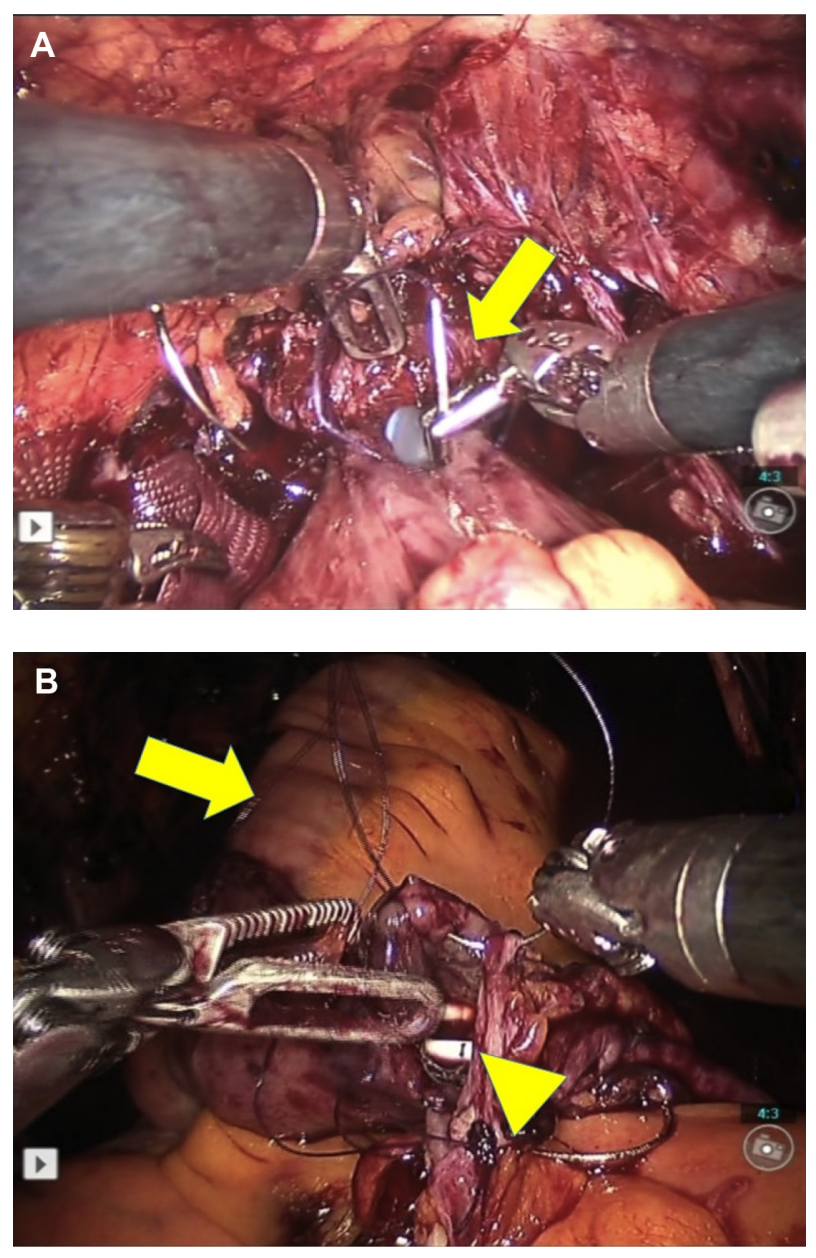

Figure 4 (A) Appearance of anastomosis between the urethra and the intracorporeal Studer pouch (arrow, please note the urethral catheter); (B) appearance of the completed intracorporeal Studer pouch. Arrow: Studer pouch, arrowhead: please note the double-J stents.

Note: Images from the archive of M Derya Balbay.

In another recent publication by Goh et al, robotic intracorporeal urinary diversion was successfully performed in 15 patients (neobladder: eight patients, ileal conduit: seven patients). In the neobladder group, the median estimated blood loss was $225 \mathrm{~mL}$, median time to regular diet was 5 days, and the median hospital stay was 8 days. Thirty- and 90-day complications were Clavien grade $1-2(\mathrm{n}=5$ and $\mathrm{n}=0)$, Clavien grade $3-5(n=2$ and $n=1)$, respectively. ${ }^{48}$

Pruthi et al reported the outcomes of twelve cases for which they performed RARC and intracorporeal urinary diversion, including ileal conduit in nine patients and orthotopic ileal neobladder in three patients. The mean operating room time was 5.3 hours and mean blood loss was $221 \mathrm{~mL}$. The mean length of hospital stay was 4.5 days. The mean time to flatus was 2.2 days and the mean time to bowel movement was 3.2 days. Overall, six complications occurred in five patients (42\%) and one was Clavien grade $\geq 3 .{ }^{49}$
Very recently, Ahmed et al ${ }^{50}$ evaluated the outcomes of intracorporeal versus extracorporeal urinary diversion following RARC by reviewing the database of the IRCC. All patients within the IRCC underwent RARC and pelvic lymph node dissection, as indicated. The urinary diversion was performed either intracorporeally or extracorporeally. Of the 935 patients who underwent RARC, 167 patients had an intracorporeal urinary diversion (ileal conduit: 106 and neobladder: 61), and 768 patients had an extracorporeal urinary diversion (ileal conduit: 570 and neobladder: 198). The operation time was similar between the groups. Although not statistically significant, the median hospital stay was found to be longer in the intracorporeal urinary diversion group ( 9 days vs 8 days; $P=0.086$ ). Reoperation rates at $0-30$ days were similar. The 90 -day complication rate was also similar between the two groups. However, a trend favoring the intracorporeal group was detected ( $41 \%$ vs $49 \% ; P=0.05$ ). Most importantly, gastrointestinal complications were significantly lower in the intracorporeal urinary diversion group $(P \leq 0.001)$. In addition, patients with intracorporeal urinary diversion were found to have a lower risk of experiencing a postoperative complication at 90 days $(32 \% ; P=0.02)$. They concluded that RARC with intracorporeal urinary diversion is a safe procedure with similar outcomes to the extracorporeal approach, with the advantage of carrying a lower risk of complications. ${ }^{50}$

In summary, the number of centers performing totally intracorporeal RARC with urinary diversion is very limited. However, interest related to this type of complex surgery is increasing, and needs further experience. Performing the whole surgery intracorporeally with the surgical robot seems to be the upper limit of the minimally invasive approach, and seems to be the future of RARC.

\section{RARC and gender, patient age, body mass index, and disease stage}

According to the published literature, RARC seems to be a safe procedure for both genders and in obese patients, ${ }^{51,52}$ although Al-Daghmin et $\mathrm{l}^{53}$ stated that female gender and obesity were independent predictors of readmission following RARC. Poch et al reported that robot-assisted intracorporeal ileal conduit could be safely performed in patients of all body mass indices. ${ }^{52}$

Richards et al evaluated the outcomes of open versus robotic RC in elderly ( $\geq 75$ years old) patients and concluded that RARC could achieve similar perioperative outcomes without compromising pathologic outcomes, with less blood loss and shorter hospital stays. ${ }^{54}$ Others also stated that RARC 
appeared to be an appropriate and safe surgical option for older patients. ${ }^{35,55}$

Regarding advanced stage bladder cancer, positive softtissue margins were reported to be similar in large open series for T2/T3 disease but inferior for bulky T4 disease. ${ }^{56}$

In summary, RARC appears to be a safe approach in elderly and obese patients, including both sexes and in advanced stage disease.

\section{Cost and RARC}

Cost is an important issue that has been discussed in some publications related to RARC. With the development and use of technology in health care such as surgical robots, increasing cost seems to be an inevitable factor that may be a concern despite the technological advantages. However, many factors are involved in cost analysis other than the surgical instruments used. Besides, the differing health care and insurance systems of various countries may also affect cost analysis.

Martin et al reported that operative time and length of stay had the greatest impact on perioperative costs following RARC and therefore concluded that RARC can provide a cost-effective alternative to open RC. They also stated that higher complication rates with open $\mathrm{RC}$ increases the total cost when compared to RARC. ${ }^{57}$ In a comparative study, Lee et $\mathrm{al}^{58}$ stated that despite a higher cost of materials, RARC was less expensive than open RC for ileal conduit and continent cutaneous diversion. The cost advantage deteriorated for orthotopic neobladder in their study. The major impact on cost was length of hospital stay. A shorter length of hospital stay was detected in the RARC group when compared to open surgery in their experience. In addition, they reported that complications materially affected cost performance. Therefore, they concluded that despite a higher cost of materials, RARC could be more cost-effective compared to open RC. ${ }^{58}$ Open RC has a cost advantage in terms of the high purchase and maintenance cost of surgical robots. However, when taking into account the inclusion of indirect costs due to complications and longer duration of hospital stay in open RC, RARC may have a cost advantage. ${ }^{59}$

Zehnder and Gill stated that cost evaluations are complex analyses influenced by many factors. In the published literature, RARC appears to be more expensive compared to open RC. Operative time, length of hospital stay, robotic maintenance and cost of the robotic instruments used, case volume, transfusion rate, and complications have been suggested to influence overall cost. ${ }^{60}$
In summary, RARC appears to have higher costs when compared to open surgery, although controversial reports exist on this issue.

\section{Conclusion}

With the increasing use of surgical robots in urologic oncology, RARC is increasingly being performed worldwide. A learning curve exists with a suggested case number of at least 20 procedures in order to reach an acceptable operation time, complication rate, and oncological outcomes including, positive SM and LN yield. Although long-term follow-up time is not available in most of the published literature, RARC seems to have acceptable oncologic and functional outcomes in addition to complication rates. Decreased intraoperative blood loss and transfusion rate are other advantages of RARC. However, some series reported decreased complication rates but increased operation time using the robotic approach. Similar oncologic outcomes in terms of positive SM rates and LN yields were reported in most comparative series. The technique of totally intracorporeal RARC including the urinary diversion is a complex procedure that is still evolving. This approach seems to have the potential of being the future of robotic RC. Cost is an important issue and RARC appears to have a higher cost when compared to the open approach.

\section{Disclosure}

Canda is a Member of Robotic Urology Working Group, Young Academic Urologists (YAU) of the European Association of Urology (EAU). The authors report no conflicts of interest in this work.

\section{References}

1. Huang GJ, Stein JP. Open radical cystectomy with lymphadenectomy remains the treatment of choice for invasive bladder cancer. Curr Opin Urol. 2007;17(5):369-375

2. Canda AE, Atmaca AF, Balbay MD. Robotic-assisted laparoscopic radical cystoprostatectomy and intracorporeal urinary diversion (Studer pouch or ileal conduit) for bladder cancer. In: Canda AE, editor. Bladder Cancer - From Basic Science to Robotic Surgery. Rijeka, Croatia: InTech; 2012:321-344.

3. Liss MA, Kader AK. Robotic-assisted laparoscopic radical cystectomy: history, techniques and outcomes. World J Urol. 2013;31(3):489-497.

4. Ahmed K, Ibrahim A, Wang TT, et al. Assessing the cost effectiveness of robotics in urological surgery - a systematic review. BJU Int. 2012;110(10):1544-1556.

5. Hayn MH, Hellenthal NJ, Seixas-Mikelus SA, et al. Is patient outcome compromised during the initial experience with robot-assisted radical cystectomy? Results of 164 consecutive cases. BJU Int. 2011;108(6):882-887.

6. Hayn MH, Hussain A, Mansour AM, et al. The learning curve of robotassisted radical cystectomy: results from the International Robotic Cystectomy Consortium. Eur Urol. 2010;58(2):197-202.

7. Mirheydar HS, Parsons JK. Diffusion of robotics into clinical practice in the United States: process, patient safety, learning curves, and the public health. World J Urol. 2013;31(3):455-461. 
8. Herr H, Lee C, Chang S, Lerner S; Bladder Cancer Collaborative Group. Standardization of radical cystectomy and pelvic lymph node dissection for bladder cancer: a collaborative group report. $J$ Urol. 2004;171(5):1823-1828; discussion 1827-1828.

9. Skinner EC, Stein JP, Skinner DG. Surgical benchmarks for the treatment of invasive bladder cancer. Urol Oncol. 2007;25(1):66-71.

10. Stein JP, Cai J, Groshen S, Skinner DG. Risk factors for patients with pelvic lymph node metastases following radical cystectomy with en bloc pelvic lymphadenectomy: concept of lymph node density. J Urol. 2003;170(1):35-41.

11. Leissner J, Hohenfellner R, Thüroff JW, Wolf HK. Lymphadenectomy in patients with transitional cell carcinoma of the urinary bladder; significance for staging and prognosis. BJU Int. 2000;85(7):817-823.

12. Herr HW, Faulkner JR, Grossman HB, et al. Surgical factors influence bladder cancer outcomes: a cooperative group report. J Clin Oncol. 2004;22(14):2781-2789.

13. Dotan ZA, Kavanagh K, Yossepowitch O, et al. Positive surgical margins in soft tissue following radical cystectomy for bladder cancer and cancer specific survival. J Urol. 2007;178(6):2308-2312; discussion 2313.

14. Linder BJ, Boorjian SA, Hudolin T, et al. Late recurrence after radical cystectomy: patterns, risk factors and outcomes. J Urol. 2013;191(5): $1256-1261$.

15. Tyritzis SI, Hosseini A, Collins J, et al. Oncologic, functional, and complications outcomes of robot-assisted radical cystectomy with totally intracorporeal neobladder diversion. Eur Urol. 2013;64(5):734-741.

16. Xylinas E, Green DA, Otto B, et al. Robotic-assisted radical cystectomy with extracorporeal urinary diversion for urothelial carcinoma of the bladder: analysis of complications and oncologic outcomes in 175 patients with a median follow-up of 3 years. Urology. 2013;82(6): 1323-1329.

17. Treiyer A, Saar M, Butow Z, Kamradt J, Siemer S, Stockle M. Robotic-assisted laparoscopic radical cystectomy: surgical and oncological outcomes. Int Braz J Urol. 2012;38(3):324-329.

18. Canda AE, Atmaca AF, Altinova S, Akbulut Z, Balbay MD. Robot-assisted nerve-sparing radical cystectomy with bilateral extended pelvic lymph node dissection (PLND) and intracorporeal urinary diversion for bladder cancer: initial experience in 27 cases. BJU Int. 2012;110(3):434-444.

19. Hellenthal NJ, Hussain A, Andrews PE, et al. Lymphadenectomy at the time of robot-assisted radical cystectomy: results from the International Robotic Cystectomy Consortium. BJU Int. 2011;107(4):642-646.

20. Hayn MH, Hellenthal NJ, Hussain A, et al. Does previous robot-assisted radical prostatectomy experience affect outcomes at robot-assisted radical cystectomy? Results from the International Robotic Cystectomy Consortium. Urology. 2010;76(5):1111-1116.

21. Khan MS, Elhage O, Challacombe B, et al. Long-term outcomes of robot-assisted radical cystectomy for bladder cancer. Eur Urol. 2013;64(2):219-224.

22. Mmeje CO, Nunez-Nateras R, Nielsen ME, et al. Oncologic outcomes for lymph node-positive urothelial carcinoma patients treated with robot assisted radical cystectomy: with mean follow-up of 3.5 years. Urol Oncol. 2013;31(8):1621-1627.

23. Menon M, Hemal AK, Tewari A, et al. Nerve-sparing robot-assisted radical cystoprostatectomy and urinary diversion. BJU Int. 2003;92(3): 232-236.

24. Torrey RR, Chan KG, Yip W, et al. Functional outcomes and complications in patients with bladder cancer undergoing roboticassisted radical cystectomy with extracorporeal Indiana pouch continent cutaneous urinary diversion. Urology. 2012;79(5):1073-1078.

25. Dindo D, Demartines N, Clavien PA. Classification of surgical complications: a new proposal with evaluation in a cohort of 6336 patients and results of a survey. Ann Surg. 2004;240(2):205-213.

26. De Nunzio C, Cindolo L, Leonardo C, et al. Analysis of radical cystectomy and urinary diversion complications with the Clavien classification system in an Italian real life cohort. Eur J Surg Oncol. 2013;39(7): 792-798.

27. Shabsigh A, Korets R, Vora KC, et al. Defining early morbidity of radical cystectomy for patients with bladder cancer using a standardized reporting methodology. Eur Urol. 2009;55(1):164-174.
28. Smith AB, Raynor M, Amling CL, et al. Multi-institutional analysis of robotic radical cystectomy for bladder cancer: perioperative outcomes and complications in 227 patients. J Laparoendosc Adv Surg Tech A. 2012;22(1):17-21.

29. Nazmy M, Yuh B, Kawachi M, et al. Early and late complications of robot-assisted radical cystectomy: a standardized analysis by urinary diversion type. J Urol. 2014;191(3):681-687.

30. Yuh BE, Nazmy M, Ruel NH, et al. Standardized analysis of frequency and severity of complications after robot-assisted radical cystectomy. Eur Urol. 2012;62(5):806-813.

31. Kauffman EC, Ng CK, Lee MM, et al. Critical analysis of complications after robotic-assisted radical cystectomy with identification of preoperative and operative risk factors. BJU Int. 2010;105(4):520-527.

32. Johar RS, Hayn MH, Stegemann AP, et al. Complications after robotassisted radical cystectomy: results from the International Robotic Cystectomy Consortium. Eur Urol. 2013;64(1):52-57.

33. Nix J, Smith A, Kurpad R, Nielsen ME, Wallen EM, Pruthi RS. Prospective randomized controlled trial of robotic versus open radical cystectomy for bladder cancer: perioperative and pathologic results. Eur Urol. 2010;57(2):196-201.

34. Parekh DJ, Messer J, Fitzgerald J, Ercole B, Svatek R. Perioperative outcomes and oncologic efficacy from a pilot prospective randomized clinical trial of open versus robotic assisted radical cystectomy. $J$ Urol. 2013;189(2):474-479.

35. Knox ML, El-Galley R, Busby JE. Robotic versus open radical cystectomy: identification of patients who benefit from the robotic approach. $J$ Endourol. 2013;27(1):40-44.

36. Styn NR, Montgomery JS, Wood DP, et al. Matched comparison of robotic-assisted and open radical cystectomy. Urology. 2012;79(6): 1303-1308.

37. Gondo T, Yoshioka K, Nakagami Y, et al. Robotic versus open radical cystectomy: prospective comparison of perioperative and pathologic outcomes in Japan. Jpn J Clin Oncol. 2012;42(7):625-631.

38. Sung HH, Ahn JS, Seo SI, et al. A comparison of early complications between open and robot-assisted radical cystectomy. J Endourol. 2012;26(6):670-675.

39. Nepple KG, Strope SA, Grubb RL 3rd, Kibel AS. Early oncologic outcomes of robotic vs open radical cystectomy for urothelial cancer. Urol Oncol. 2013;31(6):894-898.

40. Ng CK, Kauffman EC, Lee MM, et al. A comparison of postoperative complications in open versus robotic cystectomy. Eur Urol. 2010;57(2): 274-281.

41. Wang GJ, Barocas DA, Raman JD, Scherr DS. Robotic vs open radical cystectomy: prospective comparison of perioperative outcomes and pathological measures of early oncological efficacy. BJU Int. 2008;101(1):89-93.

42. Kader AK, Richards KA, Krane LS, Pettus JA, Smith JJ, Hemal AK. Robot-assisted laparoscopic vs open radical cystectomy: comparison of complications and perioperative oncological outcomes in 200 patients. BJU Int. 2013;112(4):E290-E294.

43. Sterrett S, Mammen T, Nazemi T, et al. Major urological oncological surgeries can be performed using minimally invasive robotic or laparoscopic methods with similar early perioperative outcomes compared to conventional open methods. World J Urol. 2007;25(2):193-198.

44. Laudone VP. Interim analysis of a prospective randomized trial comparing robotic and open cystectomy at Memorial SloanKettering Cancer Center. Late breaking news (Plenary Sessions). American Urological Association Annual Meeting; May 4-8, 2013; San Diego, CA.

45. Leow JJ, Reese SW, Jiang W, et al. Propensity-matched comparison of morbidity and costs of open and robot-assisted radical cystectomies: a contemporary population-based analysis in the United States. Eur Urol. Epub January 28, 2014

46. Schumacher MC, Jonsson MN, Hosseini A, et al. Surgery-related complications of robot-assisted radical cystectomy with intracorporeal urinary diversion. Urology. 2011;77(4):871-876. 
47. Atmaca AF, Canda AE, Gumus M, Asil E, Balbay MD. Delayed massive hemorrhage due to external iliac artery pseudo-aneurysm and uretero-iliac artery fistula following robotic radical cystectomy and intracorporeal Studer pouch reconstruction: endovascular management of an unusual complication. Can Urol Assoc J. 2013;7(9-10): E605-E608.

48. Goh AC, Gill IS, Lee DJ, et al. Robotic intracorporeal orthotopic ileal neobladder: replicating open surgical principles. Eur Urol. 2012;62(5): 891-901.

49. Pruthi RS, Nix J, McRackan D, et al. Robotic-assisted laparoscopic intracorporeal urinary diversion. Eur Urol. 2010;57(6):1013-1021.

50. Ahmed K, Khan SA, Hayn MH, et al. Analysis of intracorporeal compared with extracorporeal urinary diversion after robot-assisted radical cystectomy: results from the International Robotic Cystectomy Consortium. Eur Urol. 2014;65(2):340-347.

51. Butt ZM, Perlmutter AE, Piacente PM, et al. Impact of body mass index on robot-assisted radical cystectomy. JSLS. 2008;12(3):241-245.

52. Poch MA, Stegemann A, Chandrasekhar R, Hayn M, Wilding G, Guru KA. Does body mass index impact the performance of robot-assisted intracorporeal ileal conduit? J Endourol. 2012;26(7):857-860.

53. Al-Daghmin A, Aboumohamed A, Din R, et al. Readmission after robot-assisted radical cystectomy: outcomes and predictors at 90-day follow-up. Urology. 2014;83(2):350-356.

54. Richards KA, Kader AK, Otto R, Pettus JA, Smith JJ 3rd, Hemal AK. Is robot-assisted radical cystectomy justified in the elderly? A comparison of robotic versus open radical cystectomy for bladder cancer in elderly $\geq 75$ years old. J Endourol. 2012;26(10):1301-1316.
55. Coward RM, Smith A, Raynor M, Nielsen M, Wallen EM, Pruthi RS. Feasibility and outcomes of robotic-assisted laparoscopic radical cystectomy for bladder cancer in older patients. Urology. 2011;77(5): 1111-1114.

56. Challacombe BJ, Bochner BH, Dasgupta P, et al. The role of laparoscopic and robotic cystectomy in the management of muscle-invasive bladder cancer with special emphasis on cancer control and complications. Eur Urol. 2011;60(4):767-775.

57. Martin AD, Nunez RN, Castle EP. Robot-assisted radical cystectomy versus open radical cystectomy: a complete cost analysis. Urology. 2011;77(3):621-625.

58. Lee R, $\mathrm{Ng} \mathrm{CK}$, Shariat SF, et al. The economics of robotic cystectomy: cost comparison of open versus robotic cystectomy. BJU Int. 2011;108(11):1886-1892.

59. Mmeje CO, Martin AD, Nunez-Nateras R, et al. Cost analysis of open radical cystectomy versus robot-assisted radical cystectomy. Curr Urol Rep. 2013;14(1):26-31.

60. Zehnder P, Gill IS. Cost-effectiveness of open versus laparoscopic versus robotic-assisted laparoscopic cystectomy and urinary diversion. Curr Opin Urol. 2011;21(5):415-419.
Open Access Surgery

\section{Publish your work in this journal}

Open Access Surgery is an international, peer-reviewed, open access journal that focuses on all aspects of surgical procedures and interventions. Patient care around the peri-operative period and patient outcomes post surgery are key topics. All grades of surgery from minor cosmetic interventions to major surgical procedures are covered. Novel techniques

\section{Dovepress}

and the utilization of new instruments and materials, including implants and prostheses that optimize outcomes constitute major areas of interest. The manuscript management system is completely online and includes a very quick and fair peer-review system. Visit http://www.dovepress.com/ testimonials.php to read real quotes from published authors. 